International Journal of Computer Science \& Information Technology (IJCSIT) Vol 4, No 1, Feb 2012

\title{
QUALITY OF SERVICE USING PSO ALGORITHM
}

\author{
S.M. ELseuofi \\ Department of Programs, College of Community Service and Continuing Education, \\ Umm Al-Qura University \\ smseuofieuqu.edu.sa
}

\begin{abstract}
In this paper, a Particle Swarm Optimization algorithm used to solve the quality of service routing problems is proposed. Each Multicast Particle Swarm Optimization system in the Multicast Particle Swarm Optimization system router will uses an efficient objective function that reflect one or more of the QoS parameters to evaluate the multicast Tree between one source node and multiple destination nodes according to Class of Service (CoS). We first classify the applications into classes according to its sensitivity to one or more QoS parameters. Our proposed multicast PSO router finds the multicast Tree with minimum cost subject to specific QoS parameter(s) and for the specific application that belong to appropriate CoS in computer networks. The multicast PSO router system is distributed at each node in communication network and it makes its decision based on a database of alternate routes between each pairs of nodes in the network dynamically. The simulation results explain that the proposed multicast PSO systems in the Multicast Particle Swarm Optimization system router exhibits a good quality of solution and a good rate of convergence to optimal solution for each CoS that lead to high speed response in computer networks.
\end{abstract}

\section{KEYWORDS}

Multicasting, Particle Swarms Optimizer (PSO), QoS, Communication Networks, and Combinatorial Optimization.

\section{INTRODUCTION}

Multicast is a communication technique over the IP infrastructure in a network for one- tomany communication. The source sends a packet only once, even if it needs to be delivered to a large number of receivers, using the network resources optimally. The intermediate nodes replicate the packets whenever necessary to address a large receiver population [1]. The most common protocol to use multicast addressing is User Datagram Protocol (UDP). Multicast routing is used in various continuous media applications and is employed for streaming media and internet media applications. The primary function of QoS [2][6][8][9] is to ensure that all application are getting the necessary bandwidth to function at a desired level. QoS uses resource reservation control mechanisms to allow administrators to set a desired level of service for each traffic type on the network. The goal of QoS is to provide preferential delivery service for the Applications that need it by ensuring sufficient bandwidth. Controlling latency and jitter. And reducing data loss. QoS is important as it provides the following benefits, gives administrators control over network resources, ensures that time-sensitive and mission-critical applications have the resources they require, improve user experience, reduce cost by using existing resources efficiently. QoS is important for real time streaming media applications, since these often require fixed bit rate and are delay sensitive. In QoS multicast routing, each

DOI : 10.5121/ijcsit.2012.4113 
node or link has some parameters associated with it. These parameters are used to determine the most efficient path from the source to destinations. Thus these network resources must be handled and shared in such a way that the most optimal solution can be obtained for the QoS multicast routing problem with minimal cost. This cost is determined by the parameter values associated with each node and link which may be present in a chosen path from a source to a destination. Generally, heuristics are used to solve this problem. Genetic Algorithms (GA) and Ant Colony Optimization (ACO) [3] have also been to solve this problem. Particle Swarm Optimization [4][5] technique is applied to solve the QoS multicast routing problem. In this paper, we propose a Multicast Particle Swarm Optimization (PSO) Router based QoS in Communication Networks. We first classify the applications into classes according to its sensitivity to one or more QoS parameters. A multicast PSO algorithm based QoS is suggested to each class of service. The multicast PSO router finds the multicast tree with minimum cost from one source to multiple destinations subject to specific QoS parameter(s) and for the specific application that belong to appropriate class of service $(\mathrm{CoS})$ in computer networks. The multicast PSO router system is distributed at each node in communication network and it makes its decision based on a database of alternate routes between each pairs of nodes in the network dynamically. The simulation results explain that the proposed multicast PSO router exhibits a good quality of solution and a good rate of convergence to optimal solution for each $\mathrm{CoS}$ that lead to high speed response in computer networks. The remainder of the paper is organized as follows: in section 2, we review Related Works. Section 3 gives The QoS specification and Class of Service in Communication Networks, Particle Swarm Optimization, and Alternative Routes Computation. Section 4 describes the Proposed MPSOR based QoS. Simulation Results are illustrated in section 5. Conclusions and future work are drawn in section 6 .

\section{RELATED WORKS}

Many proposed intelligent algorithms with different techniques have been introduced to solve the QoS multicast routing with using one or more QoS parameters. The first class used the evolutionary algorithms is the most attractive alternative ways to go for. Zhang et al. (2008), Zhengying et al. (2001), Haghighat(2004), and Chen (2005) tackled multicast routing while looking at delay and bandwidth constraints. Roy and Das (2004) investigated multicast QoS routing to mobile phones for multimedia applications using a genetic algorithm. Simulation showed that the algorithm worked even with imprecise information. Wang et. al. (2003), Bao et al. (2006), Sun and Li (2004), and Yuan and Yan (2004) were researched multicast routing with QoS requirements using genetic algorithms. Li et. al. (2003), Tsai et. al.(2004), and Cui et. al. (2003) also investigated QoS multicast routing with genetic algorithms under various circumstances. Xu and Chen (2006) proposed an effective algorithm for solving the multicast problem with one QoS constraints. Wang et al. (2006) proposed three algorithms to construct multicast trees, which not only utilize network resources with optimal cost but also satisfy the QoS requirements of multimedia applications. These algorithms are based on three intelligent computational methods - GA, SA, and TS, respectively. There is no paper from the above class of methods used the concept of Class of Service $(\mathrm{CoS})$ in its method to support all traffic flows in the Internet. The second class of methods used the swarm intelligent methods for solving the QoS problem, Pinto and Barán (2005), Wang et al. (2009), Wang and Zhang (2005), Li and Tian (2008), Gong et al. (2007a), and Gong et al. (2007b) tackled the QoS multicast routing by using Ant colony algorithms under two or more of QoS constraints, but their works don't support the all types of traffic in the network (i.e., CoS). LIU et al. (2006) 
International Journal of Computer Science \& Information Technology (IJCSIT) Vol 4, No 1, Feb 2012

proposes PSO algorithm to solve the QoS multicast routing. The QoS multicast routing problem was transformed into a quasi-continuous problem by constructing a new integer coding and the constrained conditions in the problem were solved by the method of penalty function. SUN et al. (2006) proposes quantum PSO algorithm for solving the QoS multicast routing by converting it into an integer programming problem and then solve it by QPSO. Wang et al (2007) used the PSO to solve the bandwidth-delay constrained least cost multicast routing problem. Jin et al. (2008) proposed a novel probability convergence based particle swarm optimization algorithm for the multiple constrained QoS multicast routing. This algorithm is inspired from the probability convergence attributes. The main contents of this paper includes: (1) A novel particle sorting rule of swarm are designed. (2)A novel probability convergence mechanism is developed in the position updating phase. (3) A new anticongestion tactic is introduced. Li et al. (2007) presented a hybrid intelligent QoS multicast routing algorithm based on PSO and GA and take into account the QoS parameters (such as bandwidth, delay, delay jitter, and error rate). The above papers don't support the concept of $\mathrm{CoS}$ in the communication networks. However, we have designed a new system that supports all $\mathrm{CoS}$ in the communication networks, which is different from those multicasting methods, to support all applications in the Internet.

\section{Preliminaries}

\subsection{The QoS specification and Class of Service in Communication Networks:}

One of the most important steps in requesting QoS in communication networks is to specify what these requirements are and to quantify them accurately (QoS specifications) (Alkahtani et al., 2003). A stream of packets from a source to a destination is called a flow. In a connectionoriented network, all the packets belonging to a flow follow the same route; in a connectionless network, they may follow different routes. The needs of each flow can be characterized by four primary parameters (Tanenbaum, 2003; Forouzan, 2007): reliability, delay, jitter (delay variation), and bandwidth. We can add the security as another important and primary parameter for certain traffics such as money transactions in e-commerce, confidential or extremelyprivate applications (Alkahtani et al., 2003). Together these determine the QoS (Quality of Service) the flow requires. Several common applications and the stringency of their QoS requirements are listed in Table 1(Tanenbaum, 2003; Alkahtani et al., 2003).

Table 1: Examples of common applications and the sensitivity of their QoS requirements.

\begin{tabular}{|c|c|c|c|c|c|c|}
\hline \multirow{2}{*}{\multicolumn{2}{|c|}{ Applications }} & \multicolumn{5}{|c|}{ Sensitivity } \\
\hline & & Reliability & Delay & Jitter & Bandwidth & Security \\
\hline \multirow{4}{*}{$\begin{array}{l}\text { Data } \\
\text { traffic }\end{array}$} & E-Mail & High & Low & Low & Low & Low \\
\hline & Confidential E-Mail & High & Low & Low & Low & High \\
\hline & File Transfer & High & Low & Low & Medium & Low \\
\hline & Money Transactions & High & Low & Low & Low & High \\
\hline \multirow{5}{*}{$\begin{array}{l}\text { Real-time } \\
\text { traffic }\end{array}$} & Audio on demand & Low & Low & High & Medium & Low \\
\hline & Video on demand & Low & Low & High & High & Low \\
\hline & Telephony & Low & High & High & Low & Low \\
\hline & Videoconferencing & Low & High & High & High & Low \\
\hline & $\begin{array}{c}\text { Confidential } \\
\text { Videoconferencing }\end{array}$ & Low & High & High & High & High \\
\hline
\end{tabular}


International Journal of Computer Science \& Information Technology (IJCSIT) Vol 4, No 1, Feb 2012

From the above table 1 we suggest to classify the applications according to its Sensitivity to QoS parameter(s) into groups called Class of Service $(\mathrm{CoS})$ as in table 2.

Table 2: The groups of Applications in the CoS

\begin{tabular}{|c|c|c|}
\hline CoS & The Groups of Applications & $\begin{array}{c}\text { Sensitive to the following QoS } \\
\text { parameter(s) }\end{array}$ \\
\hline 1 & Confidential E-Mail ; Money Transactions & Reliability; Security \\
\hline 2 & E-Mail & Reliability \\
\hline 3 & File Transfer & Reliability; Bandwidth \\
\hline 4 & Audio on demand; Video on demand & Jitter; Bandwidth \\
\hline 5 & Telephony & Delay; Jitter \\
\hline 6 & Videoconferencing & Delay; Jitter; Bandwidth \\
\hline 7 & Confidential Videoconferencing & Delay; Jitter; Bandwidth; Security \\
\hline
\end{tabular}

According to above table 2 the multicast PSO router based QoS will contain seven Multicast PSO algorithms, one for each class of service $\mathrm{CoS}$ that take into account the sensitivity of its applications to the certain QoS parameter(s).

\subsection{Particle Swarm Optimization:}

Particle swarm optimization (PSO) is a stochastic optimization approach, modeled on the social behavior of bird flocks. PSO is a population-based search procedure where the individuals, referred to as particles, are grouped into a swarm that developed by Kennedy and Eberhart (Kennedy and Eberhart, 2001; Engelbrecht, 2007). Each particle in the swarm represents a candidate solution to the optimization problem. In a PSO system, each particle is "flown" through the multidimensional search space, adjusting its position in search space according to its own experience and that of neighboring particles. A particle therefore makes use of the best position encountered by itself and the best position of its neighbors to position itself toward an optimum solution. The effect is that particles "fly" toward an optimum, while still searching a wide area around the current best solution. The performance of each particle (i.e. the "closeness" of a particle to the global minimum) is measured according to a predefined fitness Function which is related to the problem being solved. PSO has some advantages over other similar optimization techniques such as GA, namely the following. 1) PSO is easier to implement and there are fewer parameters to adjust(Kang et al.,2008; Valle et al., 2008).2) In PSO, every particle remembers its own previous best value as well as the neighborhood best; therefore, it has a more effective memory capability than the GA (Valle et al., 2008). 3) PSO is more efficient in maintaining the diversity of the swarm (Engelbrecht, 2006; Valle et al., 2008) (more similar to the ideal social interaction in a community), since all the particles use the information related to the most successful particle in order to improve themselves, whereas in GA, the worse solutions are discarded and only the good ones are saved; therefore, in GA the population evolves around a subset of the best individuals.

\subsection{Alternative Routes Computation:}

We must first determine the all alternative routes between each Source-Destination (SD) pairs in computer network. We used the algorithm that proposed in (Idrees, 2010) for generating all paths between each two nodes in the grid network. We can also use the algorithms suggested by (Feng, 2001). The cost, delay, delay Jitter, packet loss rate, security rate and bandwidth between each two nodes can be generated randomly. This algorithm will be executed at each 
International Journal of Computer Science \& Information Technology (IJCSIT) Vol 4, No 1, Feb 2012

router in the network and only during the network configuration or changing the network topology to generate all routes between each two nodes in the network. The generated routes will be saved in a database of alternative routes for each $\mathrm{CoS}$ to be used later by the MPSO systems in the MPSOR.

\section{Proposed Algorithm}

Input

Directed acyclic graph to represent the network parameters of nodes and edges.

Output

The most optimal solution determined using the fitness function. A multicast tree representing the optimal solution.

Step 1: Initializing the system

Step 2: Computing all Paths from source to all destinations

Step 3: For I=1 to ITER:

Step 4: generating particles

Step 5: Applying fitness function

Step 6: For all particles in the system

Step 7: Updating personal best and global best values

Step 8: Obtaining new particles from existing particles based on personal best and global best values.

Step 9: END FOR

Step 10: END FOR

\subsection{Algorithm Details:}

\section{A. Computing paths from source to all destinations:}

After obtaining the input graph, we compute all possible paths from the chosen source node to each of the chosen destination nodes by applying Depth First Search(DFS) recursively. These paths are stored in vectors and used for later implementation.

B. Generating Particles : 
International Journal of Computer Science \& Information Technology (IJCSIT) Vol 4, No 1, Feb 2012

One path is chosen randomly from each node and is used to form a tree, this constitutes a particle [7]. The numbers of particles are chosen depending on the size of the input.

\section{Fitness Function:}

In this method, the fitness function initially used is:

$$
\mathrm{F}(\mathrm{x})=\mathrm{w}_{1} / \mathrm{tbw} *\left(\mathrm{w}_{2} * \operatorname{tdel}+\mathrm{w}_{3} * \operatorname{tdel}_{\mathrm{jit}}+\mathrm{w}_{4} * \mathrm{tp}_{\text {loss }}\right)
$$

Where, $\mathrm{w}_{1}, \mathrm{w}_{2}, \mathrm{w}_{3}, \mathrm{w}_{4}$ are constants

Tdel is total delay

Tdel $_{\text {jit }}$ is total delay jitter

$\mathrm{Tp}_{\text {loss }}$ is total packet loss

Tbw is total bandwidth

Queuing delay and buffer size are also included as parameters and the new fitness function thus obtained is:

$\mathrm{F}(\mathrm{x})=\mathrm{w}_{1} / \mathrm{tbw} * \mathrm{w}_{2} / \mathrm{tbs} *\left(\mathrm{w}_{3} *\right.$ tdel $\left.+\mathrm{w}_{4} * \mathrm{tdel}_{\mathrm{jit}}+\mathrm{w}_{5} * \mathrm{tp}_{\text {loss }}+\mathrm{w}_{6} * \mathrm{tq}_{\mathrm{del}}\right)$

Here, $\mathrm{w}_{5}, \mathrm{w}_{6}$ are constants

Tbs is total buffer size

$\mathrm{Tq}_{\mathrm{del}}$ is total queuing delay

The fitness function used is minimization function.

The parameters for each tree are computed as shown in the network model description. These values are then substituted in the fitness function.

\section{Updating Personal Best and Global Best values:}

Every particle in the system has a personal best value associated with it, pbest. This pbest value represents the best fitness function value obtained by the particle when applied to the fitness function. Also, a global best value called gbest is used to store the best fitness function value obtained frim among the pbest values of all particles in the system. The personal best of each particle is compared with the value returned by the fitness function. If the fitness function value is lesser, then the personal best of the particle is set to the fitness value. When the personal best value of each particle is computed, the global best value is updated as the value of all the personal best values.

\section{E. Obtaining new particles:}

From the initial particles, new particles are obtained taking into consideration the personal best and global best values. A velocity value is associated with each particle, which is calculated as

$\mathrm{V}_{\text {new }}=\mathrm{w}^{*} \mathrm{v}_{\text {old }}+\mathrm{c}_{1} * \operatorname{rand}() *\left(\mathrm{p}_{\text {best }}-\right.$ curr $)+\mathrm{c}_{2} * \operatorname{rand}() *\left(\mathrm{~g}_{\text {best }}-\right.$ curr $)$

Where, $\mathrm{w}, \mathrm{c}_{1}, \mathrm{c}_{2}$ are constants

$\mathrm{V}_{\text {old }}$ is old value of velocity

Rand() generates random value 
International Journal of Computer Science \& Information Technology (IJCSIT) Vol 4, No 1, Feb 2012

$\mathrm{P}_{\text {best }}$ is personal best of a particle

$\mathrm{G}_{\text {bes }} \mathrm{t}$ is global best

Curr is current particle value

New particle is computed as

$$
\mathrm{X}_{\text {new }}=\mathrm{X}_{\text {old }}+\mathrm{V}
$$

The process is then applied to these new particles. This process is repeated is repeated until all particles converge towards the global best value. Thus a tree is obtained as the result. This tree represents the most efficient network between the source and given destination.

\section{Simulation ReSUlts:}

In this section, the proposed Multicast Particle Swarm Optimization Router (MPSOR) based QoS that consists of seven of Multicast PSO based QoS systems; one for each CoS is simulated on a network consists of 9-Routers to test its performance. The network example that used in this paper is illustrated in figure (2), the all edges are labeled with (cost, delay, bandwidth,

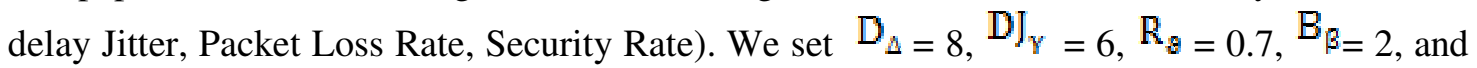

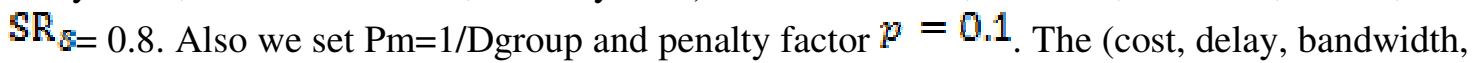
delay Jitter, Packet Loss Rate, Security Rate) on edge (i, j) is the same as with (j, i).

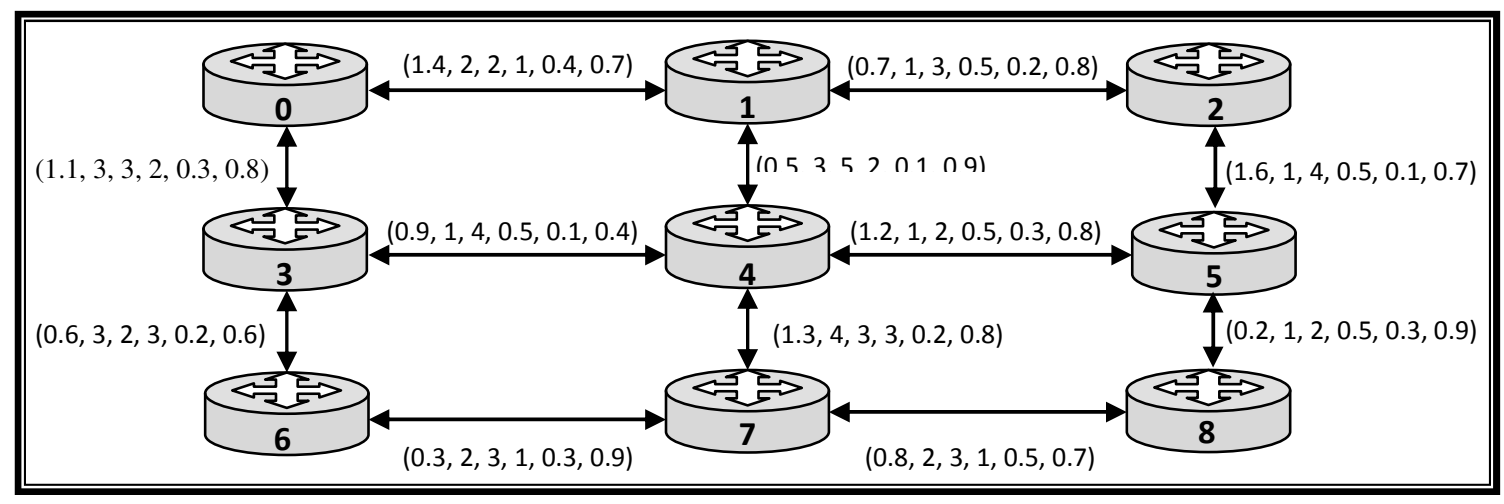

Figure (3): 9-Routers computer network example.

By using one of the algorithms in section 3.3, we can obtain for each SD pair in the network in figure (3) on the all possible routes and then stored in a database of multicast routing tables to be used later by each of the seven MPSO systems according to CoS for selecting the optimal multicast routes that satisfy the QoS parameters for sending the packet from the source router to the destination routers set. These experimental simulations are achieved by using Visual Basic 2008 professional edition on Dell laptop 1525 with processor T8300 $2.4 \mathrm{GHz}$ Core 2 due and RAM 2GB on Windows Vista Ultimate. By the simulation, many experiments will be made to explain the performance of the proposed MPSOR for QoS multicast routing.

Our performance metric measures include the Average number of Iteration of each of the seven MPSO systems (AVGITR), the Optimality of the Multicast Tree (OMT) that satisfies the QoS 
International Journal of Computer Science \& Information Technology (IJCSIT) Vol 4, No 1, Feb 2012

constraints according to $\mathrm{CoS}$, Multicast tree cost, convergence rate, and the execution time. The AVGITR and the OMT are calculated by using the following relations:

$$
\begin{aligned}
& \text { AVGITR }=\frac{\sum_{i=1}^{100} \operatorname{Itr}_{\mathrm{L}}}{100} \\
& \text { OMT }=\frac{\text { OMTN }}{100} * 100
\end{aligned}
$$

Where ${ }^{I t r_{i}}$ : the maximum number of iteration that needed by MPSO to converge to optimal solution in the ith run. $O M T N$ : The number of convergence of MPSO to optimal multicast routes that satisfy the QoS constraints according to the CoS after running it 100 times.

\subsection{The impact of the number of particles on the AVGITR and OMT:}

In this experiment, we study the impact of the number of particles on the AVGITR and the OMT for each MPSO in MPSOR. We set the Dgroup to 4. Figures 4 and 5, shows the effect of the number of particles on the AVGITR and the OMT for each MPSO in MPSOR respectively.

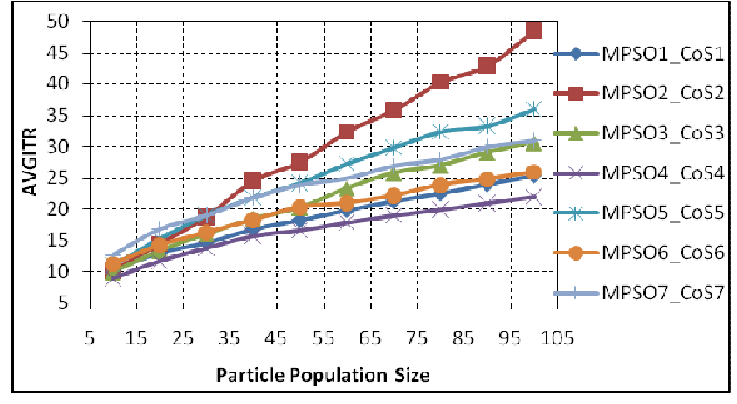

Figure (4): The impact of the number of particles on the AVGITR for each MPSO system in the MPSOR.

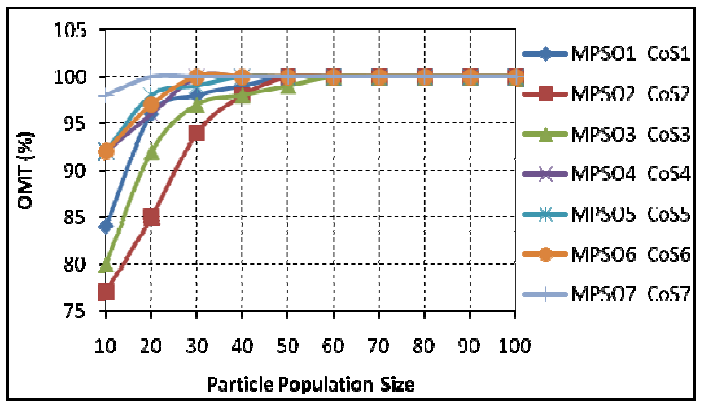

Figure (5): The impact of the number of particles on the OMT for each MPSO system in the MPSOR.

From simulation results, we see when the particle population size increase, this leads to increase each of the AVGITR and the OMT. We must make a good balance between the AVGITR and the OMT by taking the particle population size that give optimal solution with minimum AVGITR.

\subsection{The impact of Multicast Group Size on the AVGITR:}

In this experiment, we study the effect of the number of the destination nodes in the multicast group on the AVGITR of each the seven MPSO systems in MPSOR. We set the particle population size for each of MPSO1,..., MPSO7 in MPSOR to 50, 50, 60, 30, 40, 30, and 20 respectively. The source node and the destination set nodes will be selected randomly according to the network in the fig. 3. Figure 6 shows the effect of the multicast group size on the AVGITR for each of the seven MPSO systems in MPSOR.

From simulation results, we see that each of the MPSO systems in the MPSOR give optimal multicast tree that satisfy the QoS constraints according to CoS with acceptable AVGITR for each, as well as the increasing in the multicast group size may not leads to increase the AVGITR, this show the powerful performance of each of MPSO systems in MPSOR. 
International Journal of Computer Science \& Information Technology (IJCSIT) Vol 4, No 1, Feb 2012

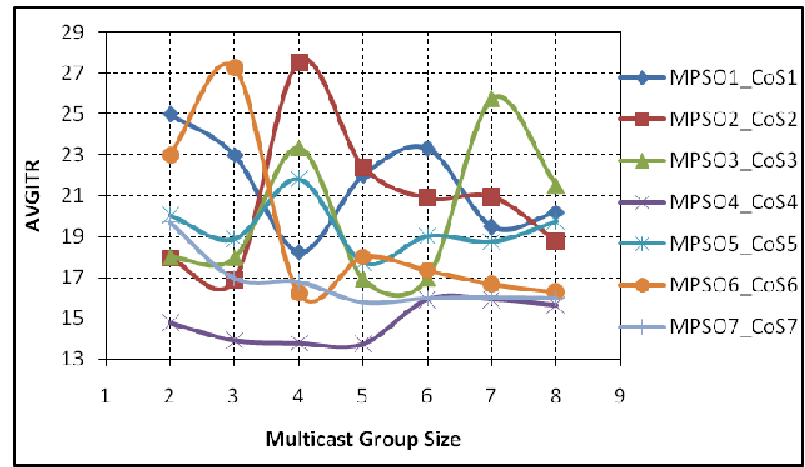

Figure (6): The impact of the multicast group size on the AVGITR for each of the seven MPSO systems in MPSOR

\subsection{The impact of Multicast Group Size on the Multicast Tree Cost:}

In this experiment, we study the effect of the number of the destination nodes in the multicast group on the Multicast Tree Cost of each the seven MPSO systems in MPSOR. We set the particle population size for each of MPSO systems in MPSOR as in experiment in section 5.2. Fig. 7 shows the Multicast Tree Cost versus the Multicast Group Size.

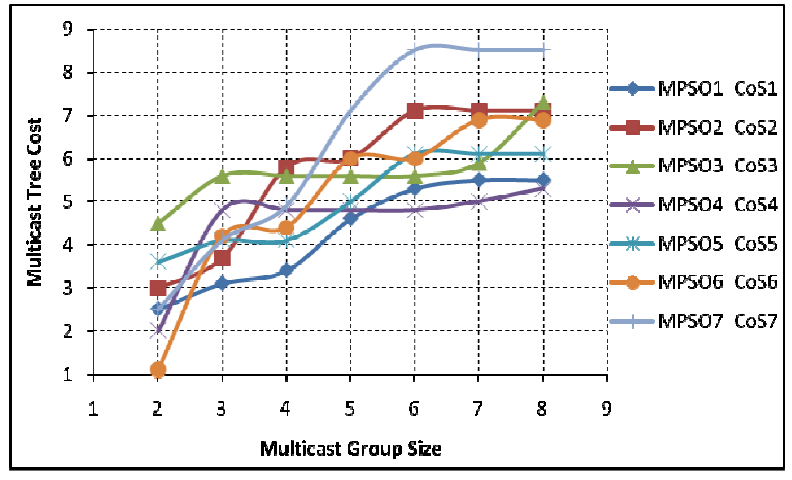

Figure (7): The Multicast Tree Cost versus the Multicast Group Size for each of the seven MPSO systems in MPSOR

From simulation results, we see that each of the MPSO systems in the MPSOR give optimal multicast tree that satisfy the QoS constraints according to CoS with minimum cost for each, but my MPSO systems in the MPSOR can achieves better optimal tree cost in both small and large multicast group size.

\subsection{The impact of Multicast Group Size on the required Execution Time:}

In this experiment, we study the effect of the number of the destination nodes in the multicast group on the execution time of each the seven MPSO systems in MPSOR. We set the particle population size for each of MPSO systems in MPSOR as in experiment in section 5.2. Fig. 8 shows the impact of the Multicast Group Size on the execution time for each of MPSO systems in MPSOR. 


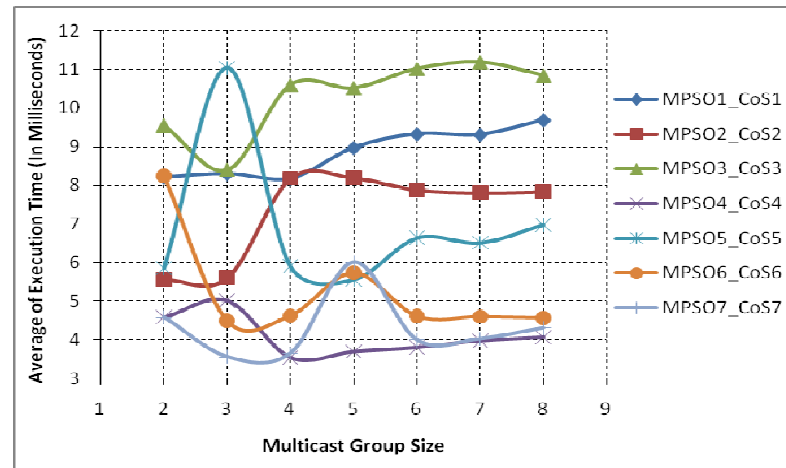

Figure (8): The impact of the Multicast Group Size on the execution time for each of MPSO systems in MPSOR

From the simulation results, we see whenever increasing the multicast group size this leads to increase or decrease the execution time that needed by each of MPSO systems in MPSOR to give optimal solution that satisfy the QoS constraints and according to the CoS. The increase in the group size that not make increase in the execution time this give additional advantage to the performance of the proposed MPSO systems in MPSOR because its high speed convergence to find the minimum cost Multicast tree that satisfy the QoS constraints and according to the appropriate $\mathrm{CoS}$.

\section{CONClusions AND Future Work:}

The simulation results show that the proposed MPSO systems in MPSOR for QoS multicast routing based $\mathrm{CoS}$ can quickly converge to optimal decision that satisfy the QoS constraints and according to $\mathrm{CoS}$ based on alternative routes in multicast routing tables that was created during the first stage of the network configuration. By using this architecture for MPSOR QoS multicasting, it can also adapt to the dynamically changing network environment such as congestion or router failure. The MPSOR will operate the appropriate MPSO system to give the QoS multicast tree according to the $\mathrm{CoS}$ that will determined by Multicast network manager. Whenever increase the Particle population size leads to increase the OMT and the AVGITR. The proposed mutation operator and the repair function that used in the proposed MPSO systems in MPSOR based QoS multicasting contribute in high speed convergence to optimal QoS multicast tree from source node to the destination node set in multicast group. The increase in the multicast group size cause increasing or decrease the AVGITR of each MPSO system in MPSOR but in acceptance rate that show the efficiency of the proposed MPSO systems in MPSOR that don't effected by the increase in the group. The proposed MPSO systems in MPSOR can achieve better optimal tree cost that satisfies the QoS constraints according to the $\mathrm{CoS}$ in both small and large multicast group size. Our proposed MPSO systems in MPSOR based QoS multicasting takes less execution time to converge to optimal solution since it uses the alternative routes which was created during the first stage of our proposed system. Our future study is to combine the proposed MPSO systems in MPSOR with other functions such as admission control and packet scheduling and classification in the design of the QoS multicast router and evaluate the performance of the router and focus on other networks such as wireless and mobile networks. 
International Journal of Computer Science \& Information Technology (IJCSIT) Vol 4, No 1, Feb 2012

\section{References}

[1] ZiqiangWang, Xia Sun, Dexian Zhang, “ A PSO Based Multicast Routing Algorithm, “ IEEE Third International Conference on Natural Computation(ICNC),pp,664-667, 2007.

[2] Liu Jing, Jung Sun, Wenbo Xu, "QOS Multicast Routing based on Particle Swarm Optimization: E.Cochado et al.(Eds,): IDEL LNCS 4224 , pp. 936-943, 2006

[3] Wang, x., Zou, R., and Huang, M. (2009). An ABC Supported QoS Multicast Routing Scheme Based on Ant Algorithm, 2009 International Conference on Communications and Mobile Computing, January 06-January 08,vol. 3, pp. 222-226.

[4] Zhang, O. Wang, Z., and Zhang, D. (2008). Qos Multicast Routing Optimization Based on Memetic Algorithm, International Conference on Management of e-Commerce and eGovernment, October 17-October 19, p. 441-444.

[5] Zhang, L., Cai, L., Li, M., and Wang, F.(2009). A method for least-cost QoS multicast routing based on genetic simulated annealing algorithm, Computer Communications Vol. 32, No. 1, pp. $105-110$.

[6] Xing, H., Ji , Y., Bai, L., Liu, X., Qu, Z., and Wang, X. (2009). An adaptive-evolution-based quantum-inspired evolutionary algorithm for QoS multicasting in IP/DWDM networks, Computer Communications, Vol. 32 , Issue 6, pp. 1086-1094.

[7] Xing,H., Liu,X., Jin,X., Bai,L., and Ji, Y.(2009). A multi-granularity evolution based Quantum Genetic Algorithm for QoS multicast routing problem in WDM networks, Computer Communications, Vol. 32, issue 2, pp. 386-393.

[8] Vijayalakshmi, K. and Radhakrishnan, S. (2008a).Dynamic Routing to Multiple Destinations in IP Networks using Hybrid Genetic Algorithm (DRHGA), International Journal of Computer Science Vol. 4, No. 1, pp. 43-52.

[9] Valle,Y., Venayagamoorthy, G. K., Mohagheghi, S., Hernandez,J. and Harley, R. G. (2008). Particle Swarm Optimization: Basic Concepts, Variants and Applications in Power Systems, IEEE TRANSACTIONS ON EVOLUTIONARY COMPUTATION, vol. 12, no. 2, pp. 171195.

\section{Author}

S.M. ELseuofi $\mathrm{He}$ is a lecturer at Department of Programs College of Community Service and Continuing Education, Umm Al-Qura University, Saudi Arabia. His current research interests include Communication Networks, Data mining, Text Classification, E-mail Classification and Spam Management using AI techniques.

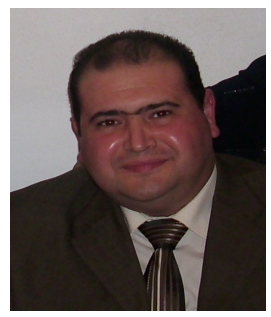

